\title{
Stem cells derived from testis show promise for treating a wide variety of medical conditions
}

\author{
Karim Nayernia ${ }^{1}$ \\ ${ }^{I}$ North East England Stem Cell Institute (NESCI) and Institute of Human Genetics, University of Newcastle upon Tyne, International \\ Centre For Life, Central Parkway, Newcastle upon Tyne, NE1 3BZ, UK. Karim.nayernia@newcastle.ac.uk
}

Cell Research (2007) 17:895-897. doi: 10.1038/cr.2007.96; published online 15 November 2007

The continuation of the spermatogenic process throughout life relies on a proper regulation of self-renewal and differentiation of germline testis stem cells, the spermatogonial stem cells. These are single cells situated on the basal membrane of the seminiferous epithelium. Only $0.03 \%$ of all germ cells are spermatogonial stem cells (SSCs) [1-3]. To maintain spermatogenesis, the processes of self-renewal and differentiation of SSCs must be precisely regulated by intrinsic gene expression in the stem cells and extrinsic signals, including soluble factors or adhesion molecules from the surrounding microenvironment, the stem cell niche.

Spermatogonial stem cells are descendants of the primordial germ cells (PGCs), which migrate from extraembryonic sites to colonize the gonadal ridge early during embryonic life. In the females, the primordial germ cells proliferate extensively and enter meiotic prophase at around the time of birth. In males, cessation of germ cell proliferation and blockade of meiotic entry are important steps in the morphogenetic cascade initiated by the expression of the SRY gene. The male germ cells associate with somatic cells of the presumptive gonad and form testicular cords, which mark the differentiation of PGCs into gonocytes. Different stages of germ cell development to spermatogonial stem cells are shown in Figure 1.

Several lines of evidence have suggested extensive proliferation activity and pluripotency of germline stem cells, including spermatogonial stem cells.

In an early embryo a cell has the potential to generate many different cell types. During development cells generally lose this potential or 'potency', and become restricted to making one or a few cell types. Cells can become undetermined and undifferentiated in special circumstances. These undifferentiated cells which are capable of self-renewal and differentiation into more specialized cells are termed as stem cells $[4,5]$. Stem cells have the remarkable potential to develop into many different cell types in the body. Serving as a sort of repair system for the body, they can theoretically divide without limit to replenish other cells as long as the person or animal is still alive. When a stem cell divides, each new cell has the potential to either remain a stem cell or become another type of cell with a more specialized function, such as a muscle cell, a red blood cell, or a brain cell. Embryonic stem cells, as their name suggests, are derived from embryos.

There are several lines of evidence which show the potency of germ cells in prenatal and postnatal stages. Cultured PGCs exposed to a specific cocktail of growth factors give rise to embryonic germ cells, pluripotent stem cells that can contribute to all the lineages of chimeric embryos including the germline. The conversion of PGCs into pluripotent stem cells is a remarkably similar process to nuclear reprogramming in which a somatic nucleus is reprogrammed in the egg cytoplasm. The conversion of PGCs into pluripotent stem cells may be linked in some way with their deregulated proliferation $[6,7]$. Other evidence is the pluripotency of testicular germ cell tumors. Testicular teratomas are highly unusual benign tumors containing derivatives of the three primary germ layers. Different studies have shown that embryonal carcinomas (EC) are derived from PGCs [8]. Assays of developmental potency show that isolated EC cells are pluripotent stem cells, but when they lose the ability to differentiate they form malignant teratocarcinomas. Understanding the genetics of embryonal carcinoma cell formation and the growth factor signaling pathways controlling embryonic germ cell derivation could tell us much about the molecular controls on developmental potency in mammals $[9,10]$.

After birth, two stages of germline stem cell differentia- 
In vivo
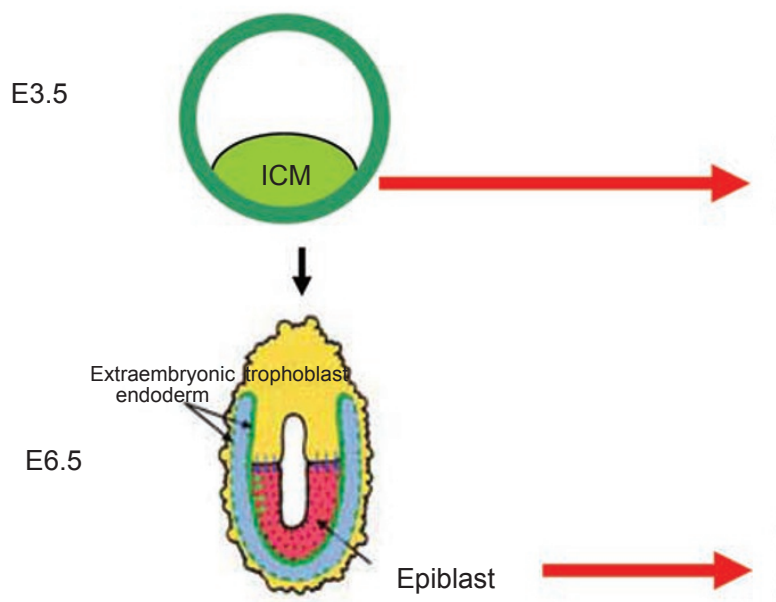

(1)

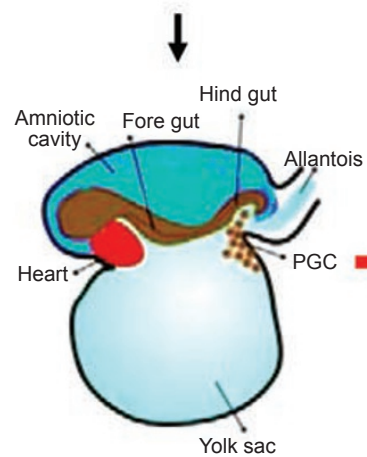

E8.5

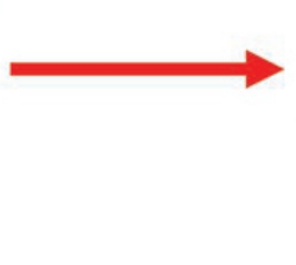

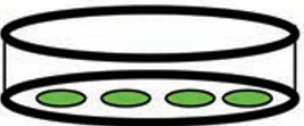

Embryonic stem cells

(ES cells)

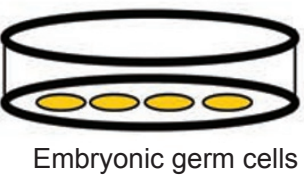
(EG cells)

Neonatal

testis
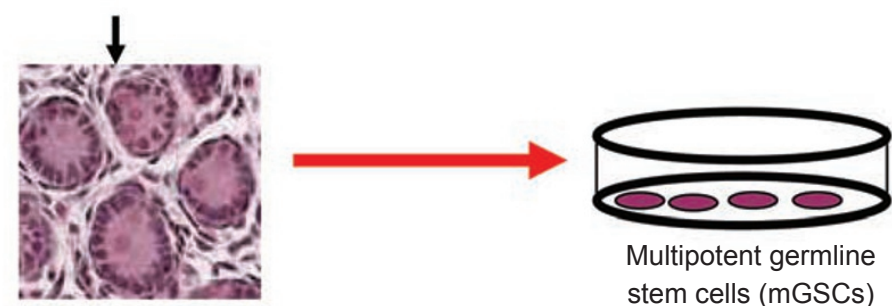

Multipotent germline stem cells (mGSCs)
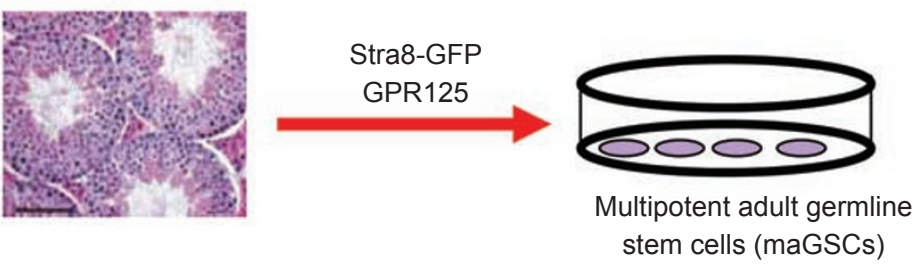

Figure 1 Different stages of germ cell development in vivo and pluripotency of germline stem cells after isolation and culture in vitro. Several lines of evidence indicate the pluripotency of germ cells in all developmental stages. ES cells: embryonic stem cells; EG cells: embryonic germ cells, mGSCs: multipotent germ line stem cells; maGSCs: multipotent adult germline stem cells, SPCs: spermatogonial progenitor cells.

tion have been discussed: neonatal and adult stages. Cells isolated from neonatal testis are able to differentiate to the cells of all three germ layers $[11,12]$, including mesodermal cells, namely cardiomyocytes and endothelial cells and therefore these cells are termed as multipotent germline stem cells (mGSCs, Figure 1). In adult, germline stem cells 
develop to spermatogonial stem cells or spermatogonial progenitor cells. Recently, two groups showed successful isolation, culture and characterization of these cells [13-15]. The first group used a genetic selection strategy using a spermatogonial specific promoter (Stra8) and green fluorescence protein (GFP) to isolate the cells from adult mouse testis. They were able to isolate and culture these cells in culture medium containing the precise combination of cellular growth factors needed for the cells to reproduce themselves in vitro [13]. These cells called multipotent adult germline stem cells (maGSCs) are pluripotent (Figure 1). More recently, the group of Shahin Rafii could confirm the pluripotency of spermatogonial stem cells and were able to isolate these cells by a specific surface maker, GPR 125 . Expression of GPR125 in the testis was detected exclusively in spermatogonia and not in differentiated germ cells [15]. They isolated GPR 125 positive cells and termed the cells as spermatogonial progenitor cells (SPCs). Stemness of SPCs was shown by testicular transplantation assay and reconstruction of spermatogenesis. GPR125+ cells generated derivatives of the three germ layers and contributed to chimaeric embryos. These cells are also able to differentiate into contractile cardiac tissue in vitro and formed functional blood vessels in vivo [15].

These data clearly indicate the pluripotency of adult germline stem cells, spermatogonial stem cells (Figure 1). These cells were characterized concerning their molecular profiling and these were compared to molecular profiling of ES cells using a stem cell array which contains relevant genes related to stem cell metabolism. The results indicate that spermatogonial stem cells share many molecular characteristics with embryonic stem cells. On cellular level, spermatogonial stem cells resemble embryonic stem cells; they form embryoid body structure after two weeks of culture. In contrast to ESCs, use of SSCs for cell transplantation will allow establishment of individual cell-based therapy, because the donor and recipient are identical. In addition, the ethical problem is avoided. These characteristics provide new and unprecedented opportunities for the therapeutic use of spermatogonial stem cells for regenerative medicine.
If researchers can isolate such cells from human, germline stem cells can be reprogrammed to have the ability to differentiate into cells that can be used therapeutically, thus could serve as a new promising source of stem cells for cell-based regenerative medicine.

\section{References}

1 Kubota H, Brinster RL. Technology insight: In vitro culture of spermatogonial stem cells and their potential therapeutic uses. Nat Clin Pract Endocrinol Metab 2006; 2:99-108.

2 Olive V, Cuzin F. The spermatogonial stem cell: from basic knowledge to transgenic technology. Int J Biochem Cell Biol 2005; 37:246-250.

3 Nayernia K, Li M, Engel W. Spermatogonial stem cells. Methods Mol Biol 2004; 253:105-120.

4 Gardner RL. Stem cells: potency, plasticity and public perception. J Anat 2002; 3:277-282.

5 Reik W, Dean W, Walter J. Epigenetic reprogramming in mammalian development. Science 2001; 293:1089-1093.

6 Cooke JE, Godin I, Ffrench-Constant C, et al. Culture and manipulation of primordial germ cells. Methods Enzymol 1993; 225:37-58.

7 McLean DJ. Vertebrate reproductive stem cells: recent insights and technological advances. Semin Cell Dev Biol 2006; 17:534539

8 Stevens LC. Origin of testicular teratomas from primordial germ cells in mice. J Natl Cancer Inst 1967; 38:549-552.

9 Damjanov I. Teratocarcinoma stem cells. Cancer Surv 1990; 2:303-319.

10 Hussain A. Germ cell tumors. Curr Opin Oncol 2005; 17:268274.

11 Kanatsu-Shinohara M, Inoue K, Lee J, et al. Generation of pluripotent stem cells from neonatal mouse testis. Cell 2004; 119:1001-1112.

12 Kanatsu-Shinohara M, Shinohara T. The germ of pluripotency. Nat Biotechnol 2006; 24:663-664.

13 Guan K, Nayernia K, Maier LS, et al. Pluripotency of spermatogonial stem cells from adult mouse testis. Nature 2006; 440:1199-1203.

14 Guan K, Wagner S, Unsold B, et al. Generation of functional cardiomyocytes from adult mouse spermatogonial stem cells. Circ Res 2007; 100:1615-1625.

15 Seandel M, James D, Shmelkov SV, et al. Generation of functional multipotent adult stem cells from GPR125+ germline progenitors. Nature 2007; 449:346-350. 